\title{
Evaluating an e-mental health program ("deprexis") as adjunctive treatment tool in psychotherapy for depression: design of a pragmatic randomized controlled trial
}

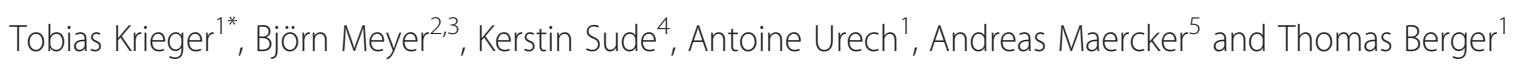

\begin{abstract}
Background: Major depressive disorder (MDD) places a significant disease burden on individuals as well as on societies. Several web-based interventions for MDD have shown to be effective in reducing depressive symptoms. However, it is not known whether web-based interventions, when used as adjunctive treatment tools to regular psychotherapy, have an additional effect compared to regular psychotherapy for depression.

Methods/design: This study is a currently recruiting pragmatic randomized controlled trial (RCT) that compares regular psychotherapy plus a web-based depression program ("deprexis") with a control condition exclusively receiving regular psychotherapy. Adults with a depressive disorder $(\mathrm{N}=800)$ will be recruited in routine secondary care from therapists over the course of their initial sessions and will then be randomized within therapists to one of the two conditions. The primary outcome is depressive symptoms measured with the Beck Depression Inventory (BDI-II) at three months post randomization. Secondary outcomes include changes on various indicators such as anxiety, somatic symptoms and quality of life. All outcomes are again assessed at the secondary endpoint six months post randomization. In addition, the working alliance and feasibility/acceptability of the treatment condition will be explored.
\end{abstract}

Discussion: This is the first randomized controlled trial to examine the feasibility/acceptability and the effectiveness of a combination of traditional face-to-face psychotherapy and web-based depression program compared to regular psychotherapeutic treatment in depressed outpatients in routine care.

Trial registration: ISRCTN20165665.

Keywords: Depression, Internet-based treatment, Adjunctive treatment tool, Randomized controlled trial (RCT)

\section{Background}

Major depressive disorders (MDD) are among the leading causes of worldwide disability [1]. Several meta-analysis have shown that web-based self-help interventions can contribute to depressive symptom reduction and there is considerable support for the use of the Internet for delivering evidence-based psychotherapy for depression [2-7]. However, several studies suggest that web-based self-help interventions without support seem to result in more

\footnotetext{
* Correspondence: tobias.krieger@psy.unibe.ch

${ }^{1}$ Department of Clinical Psychology and Psychotherapy, University of Bern, Bern, Switzerland

Full list of author information is available at the end of the article
}

modest outcomes and considerably higher dropout rates than treatments including regular web-based support from therapists or technically qualified persons [6,8-10].

There is growing knowledge about the effects of webbased interventions. However, research on combining web-based technologies with traditional face-to-face therapy, i.e., blended treatments, is sparse. Importantly, the term blended treatment includes any possible combination of regular face-to-face treatments and web-based interventions. For instance, treatment components of a web-delivered intervention may be integrated intensively and used during the face-to-face treatment, or, as in the current study, may rather be used as an adjunctive 
intervention. Since research on blended treatments is scarce, it remains to a large extent an open question if and how web-based interventions can be combined meaningfully with traditional forms of psychotherapy and whether this may have an incremental effect [11].

So far, only a few studies have investigated combinations of regular face-to-face therapy and web-based adjunctive tools in mental disorders. For example, there are promising results regarding symptomatology and acceptability for (palmtop-) computer-assisted psychotherapies in different anxiety disorders [12-14]. Furthermore, in a study in which 77 individuals seeking treatment for substance dependence were randomly assigned to regular face-to-face psychotherapy or regular face-to-face psychotherapy with biweekly access to a computer-based training in cognitive-behavioral therapy (CBT), participants in the blended condition submitted significantly more drug-negative urine specimens and tended to have longer continuous periods of abstinence during treatment [15]. Moreover, a 6-month followup showed significantly better durability of effects of the combined treatment over regular treatment for both self-report and urinalysis data [16]. These results were replicated in a larger randomized clinical trial in a more homogeneous clinical population, i.e., cocaine-dependent individuals maintained on methadone [17].

Regarding MDD and combined treatments, research is very sparse. For example, Wright and colleagues conducted an open trial of a computer-assisted psychotherapeutic treatment in a sample consisting predominantly of patients suffering from depression. Results indicated a high rate of patient acceptance of this form of treatment, and scores of symptom severity as well as the mean score on a measure of cognitive therapy knowledge were significantly improved [18]. Subsequently, Wright and colleagues [19] conducted a RCT comparing a conventional face-to-face cognitive therapy (CT), a wait-list, and computer-assisted cognitive therapy with reduced therapist contact in patients suffering from a MDD. Results indicated that the combined condition was more effective than the wait-list condition and equally effective as conventional CT. These effects were maintained over a 6-month period. Furthermore, computer-assisted cognitive therapy showed more robust effects, relative to being wait-listed, than standard cognitive therapy in reducing measures of cognitive distortion and in improving knowledge about cognitive therapy.

Recently, Mansson et al. [20] conducted a proof-ofconcept study on the effectiveness of a blended form of psychotherapy in a mixed anxious-depressed sample. The adjunctive web-based platform was to be used at home and in the clinical setting where treatment was provided. The main part of the platform consisted mainly of material that would have been presented on paper or verbally in a face-to-face session. Additionally, the platform comprised some basic components of CBT, such as scheduling sessions, keeping an agenda, and setting goals. Results indicated that this blended treatment had a positive effect across anxious and depressive symptom measures as well as on a quality of life measure in a mixed sample of 15 patients suffering from MDD and/or anxiety disorders. Additionally, patients as well as therapists rated the treatment mostly favorably. Positive patients' feedback included, for example: memory support and learning, potential to gain an overview of the treatment process, positive implications for homework assignments, promotion of a sense of autonomy and responsibility.

The literature cited above indicates that combining traditional face-to-face psychotherapy and web-based tools may be a promising venue. A possible explanation for these results is highlighted by a recent review, which concludes that patients' treatment adherence may be improved through new technologies [21]. Potential benefits of blending treatments could for example be a greater reduction of depressive symptoms or more cost-efficacious treatments [19]. However, there could also be some serious downsides of combining conventional face-to-face treatments and new technologies. For example, a recent randomized controlled study showed that appointment reminder via SMS did not lead to higher appointment attendance as expected and, more importantly, that more client dropped out in the reminder condition compared to the no reminder condition [22]. Finally, to our knowledge there is no study that investigates how the blending of treatments affects important process variables such as the therapeutic alliance.

\section{Objective and research questions}

The main objective of the present study is to further investigate combined treatment approaches for MDD by evaluating an empirically validated web-based treatment (deprexis; [23-25]) as an adjunctive tool in regular psychotherapeutic treatment in comparison with traditional psychotherapy in a sample of depressed outpatients by means of a pragmatic RCT in routine care. Therefore, our main hypothesis is that the combined treatment (TAU plus Deprexis) is more effective than regular psychotherapeutic treatment (TAU) alone.

Additionally, during a 6-month period we intend to test on an exploratory basis how helpful patients as well as therapists rate the combined treatment and whether the combination is associated with increased efficiency (i.e., reduction of number of sessions) or with negative side effects (e.g., lower quality of the therapeutic alliance, increased drop-out rate).

\section{Methods/design}

\section{Design}

A two-armed randomized pragmatic RCT is currently being conducted to compare regular face-to-face psychotherapeutic treatment with a combined treatment, 
i.e., face-to-face psychotherapeutic treatment plus a webbased adjunctive treatment tool (deprexis). Measurements in both conditions are assessed at baseline (T0), after six weeks (process measures), after three months (T1), and after six months follow-up (after randomization) (T2). The study design is shown in Figure 1.

\section{Ethics}

The study is being conducted in compliance with the Declaration of Helsinki and has been approved by the Ethics Committee of the German Psychological Association (DGPs, TB072013; 16/08/2013) and the Hamburg Chamber of Psychotherapists (18/12/2013). Participants are informed that they can withdraw at any time without having to disclose reasons. Informed consent is obtained online prior to the baseline assessment. All participants receive oral and written information from their therapist about the aim of the study, benefits and risks of participation and the study procedure.

\section{Inclusion and exclusion criteria}

We include adults (a) with age 18 and above, who (b) suffer from a MDD according to the International Classification of Diseases (ICD-10; i.e., F32.-depressive episode, F33.- Recurrent Depressive Disorder, F34.- Persistent Affective Disorder, F38.- Other Affective Disorder, F39 Unspecified Affective Disorder), (c) have a Beck Depression Inventory (BDI-II) sum score over 13, (d) have sufficient knowledge of the German language, (e) have Internet access and sufficient knowledge to use it (based on self-report), and (f) are willing to give informed consent. All participants are recruited in Germany.

We will exclude subjects who (a) have a known psychotic or bipolar disorder, (b) suffer from a chronic depression with onset in childhood (based on clinical judgment),

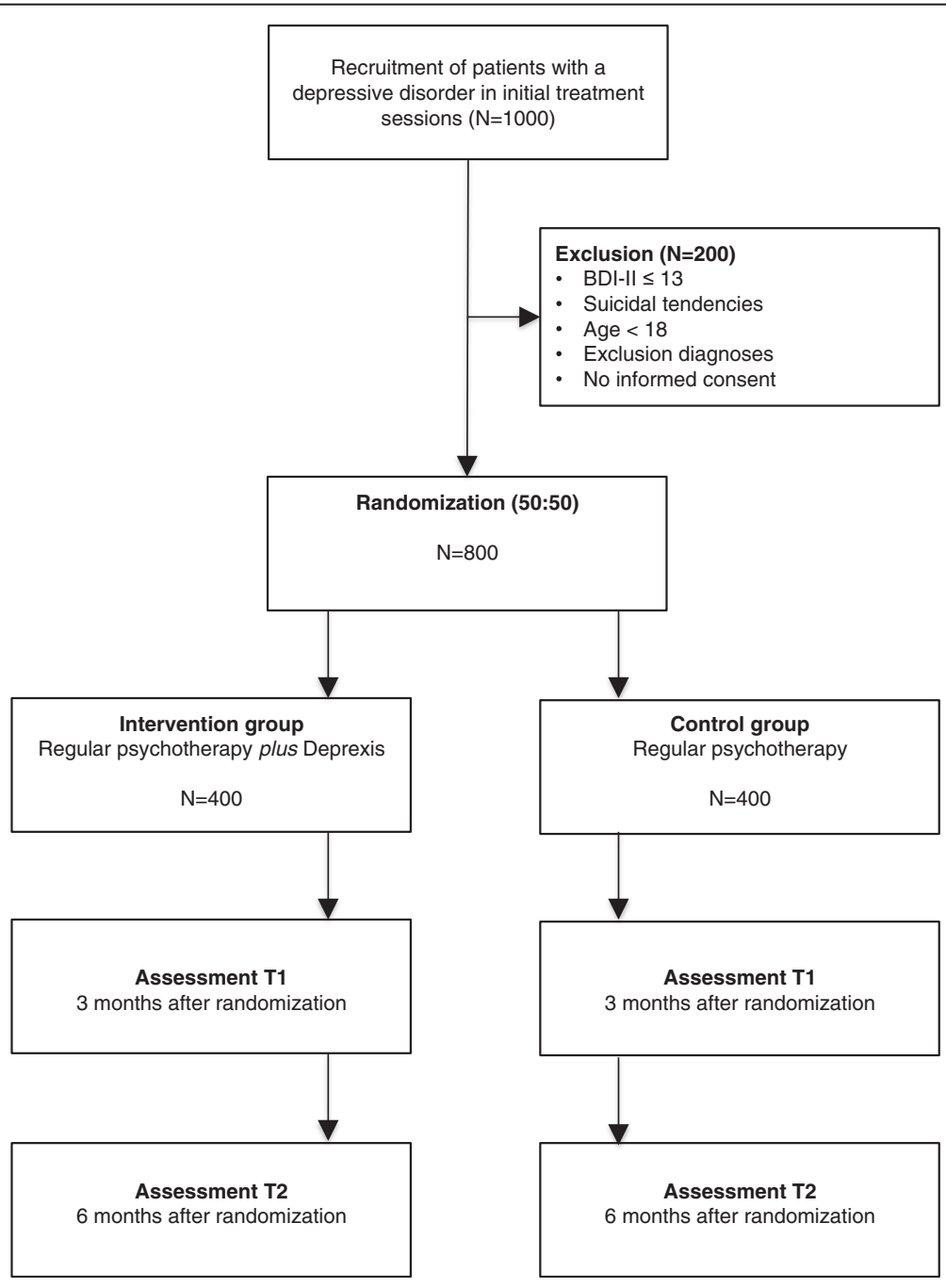

Figure 1 Study design. 
and (c) show a notable suicidal risk (based on clinical judgment of the therapists).

\section{Recruitment}

Forty therapists, all members of the Deutsche PsychotherapeutenVereinigung (DPtV), working in secondary care will be recruited. All therapists are informed about the study by the DPtV and all interested members are invited to participate in the study. Participating therapists inform their self-referred patients about the study and ask them if there are willing to take part in the present study. Potential study participants (patients) are informed about the possibility of taking part in the present study in the initial therapy session by their therapist. Interested patients receive an information sheet and a written informed consent form by the therapists and are invited to ask questions. After returning the completed informed consent form individually to the therapists, participants are asked to complete online screening questionnaires at home to assess whether they fulfill the inclusion criterion of BDI-IIScore $>13$.

\section{Randomization}

Participants who return the informed consent and meet all of the inclusion and none of the exclusion criteria will be randomly allocated to the study conditions (50:50). Randomization takes place at an individual level within therapists. Thus, therapists provide both treatments, allowing us to control for therapist effects. The allocation list will be produced using an automated computer-generated random numbers table placed in a secured web-based database and will be concealed to the investigators and the therapists (www.random.org). After randomization, the participant and his or her therapist will be informed about the condition by email.

\section{Blinding}

In the present study, there is no blinding implemented, consistent with recommendations for the conduct of pragmatic RCTs [26]. All participants will be informed about the aims and the methodology of the study. They can ask questions regarding the study and withdraw their informed consent at any time. The focus of the present study is on external validity and generalizability of the results to routine clinical practice.

\section{Description of the interventions}

Intervention group: Psychotherapy plus web-based online platform (deprexis)

Participants in the intervention group receive access to the deprexis platform. Trained, certified psychotherapists introduce the patients to the use of the program. There are no costs for patients or therapists for the program use during the study.
Deprexis provides psychoeducational information and exercises that are mainly based on cognitive-behavioral psychotherapy and aim at decreasing depressive symptoms. Deprexis includes ten modules plus one summary module: (1) psychoeducation, (2) behavioral activation, (3) cognitive restructuring, (4) mindfulness and acceptance, (5) interpersonal skills, (6) relaxation, (7) physical activity and lifestyle modification (e.g., exercise and nutrition), (8) problem-focused approaches, (9) expressive writing and schema-focused contents, (10) positive psychology as well as emotion-focused-interventions. A more comprehensive description of the program can be found in Meyer and colleagues [23].

Communication between the participant and deprexis takes place over simulated online-dialogues in which the user reacts to the content by clicking on multiple-choice answers, e.g., in order to express doubts, to confirm particular contents, or to request further information. Text units are illustrated with drawings, pictures and audio clips. The modules can be accessed repeatedly during the intervention interval. Depending on the reading speed of the participants the completion of a module takes between 10 and 60 minutes.

Furthermore, deprexis includes short questionnaires for the assessment of current mood, which offers the possibility to visually inspect the mood trajectory over the course of therapy. Additionally, the program provides the user with printable summaries and work sheets. Optionally, the user receives daily short text messages to remind him or her to use the program and reiterate program content.

Therapists can support participants with the program use following clinical judgment and discuss progress with them during face-to-face sessions. For this purpose, therapists will be introduced to the content of the program and the "therapist cockpit" in a three-hour workshop. In the therapist cockpit, therapists can track participants' program use and symptomatic progress. In the workshops, therapists are informed that the program can be regarded as an adjunctive tool that need not influence the content of their face-to-face sessions. For continuous support, participating therapists can contact the program developer and owner (GAIA AG, Hamburg) by phone or email as required.

\section{Control group: Psychotherapy without Deprexis}

Patients in the control group receive regular psychotherapy, i.e., usually weekly one-hour sessions, according to the clinical judgment of the therapists. The behavior of the therapists and the number of therapy sessions are not deliberately influenced by the study. In case of interest, participants in the control condition will receive access to deprexis after completion of the study. 


\section{Therapists}

All therapists are members of the DPtV. With over 10,000 psychotherapists the DPtV is the largest advocacy for approved psychological psychotherapists and child and adolescent psychotherapists in Germany. Most participating psychotherapists in the study identify themselves as being eclectic with a focus on cognitive-behavioral therapy, but also psycho-dynamically oriented therapists take part in the study.

\section{Sample size calculation}

Regarding differences between the two active treatment conditions, we want to be able to detect standardized between-groups effects sizes (Cohen's $d$ ) of 0.2. Smaller effect sizes are considered to be negligible from a clinical point of view [27]. At an $\alpha$ error level of .05 (two-tailed), a statistical power (1-Beta) of .80 , and a correlation between pre- and post-measurements found in our earlier studies, we need to include 800 participants. This sample size calculation is conservative and is based on a current meta-analysis in which a mean effect of $d=.28$ for online depression programs was found [5]. Since in the current study participants in both conditions will receive psychotherapy, a significant reduction of depressive symptoms can also be expected in the control condition. Therefore, a relatively small effect size between conditions, i.e., depression level after three months between deprexis plus psychotherapy vs. psychotherapy is expected.

\section{Primary and secondary outcomes}

Primary outcome is depressive symptoms assessed with the Beck Depression Inventory, second edition (BDI-II). In secondary analyses, we will explore the effects on quality of life, anxiety, somatic symptomatology, health-related quality of life, psychological aspects of the treatment process, and psychological empowerment. All measurements are assessed online. In order to promote completion when assessments are missing, participants will be automatically reminded twice to complete the assessments. For an overview of all outcome measures, see Table 1.

\section{Outcome measures}

\section{Depressive symptomatology}

The German version of the BDI-II [28] will be the primary outcome measure. The BDI-II is a widely used self-report measure for the assessment of depression severity with good psychometric properties and a completion time of 5-10 minutes. Analogous to a previous study [25] on the efficacy of online depression treatment, we only include patients with a score greater than 13 . Scores from 0-13 indicate no or minimal depressive symptoms, scores from 14-19 indicate mild depression, from 20-28 moderate depression, and from 29-63 severe depression.

\section{Web Screening Questionnaire (WSQ)}

The WSQ is a 15-item self-report instrument screening for frequent mental disorders [29]. Evidence indicating

Table 1 Outcome measures

\begin{tabular}{|c|c|c|}
\hline & Patients & Therapists \\
\hline \multirow[t]{9}{*}{ T0 (Baseline) } & - Demographic Variables Questionnaire & - Questions regarding the theoretical orientation \\
\hline & • Depression: BDI-II & \multirow[t]{8}{*}{ ICD-10 diagnoses of the patient } \\
\hline & - Suicidal tendencies: SBQ-R & \\
\hline & - Web Screening Questionnaire (WSQ) & \\
\hline & - Anxiety: GAD-7 & \\
\hline & - Somatic symptoms: PHQ-15 & \\
\hline & - Health-related quality of life: SF-12 & \\
\hline & $\begin{array}{l}\text { - Questionnaire for the evaluation of psychotherapy } \\
\text { courses (FEP-2) }\end{array}$ & \\
\hline & - Empowerment: Psychological Empowerment Scale & \\
\hline 6 weeks & Working Alliance Inventory (patient - perspective) & Working Alliance Inventory (therapist - perspective) \\
\hline 12 weeks & Working Alliance Inventory (patient - perspective) & Working Alliance Inventory (therapist - perspective) \\
\hline \multirow[t]{3}{*}{ T1 (Primary endpoint) 3 months } & As baseline, plus: & \multirow{3}{*}{$\begin{array}{l}\text { Assessment of acceptability of treatment in } \\
\text { psychotherapy }\end{array}$} \\
\hline & - Subjective rating of the treatment course & \\
\hline & In intervention group: Subjective rating of treatment & \\
\hline \multirow[t]{3}{*}{ T2 (Secondary endpoint) 6 months } & As Baseline, plus: & \multirow{3}{*}{$\begin{array}{l}\text { Assessment of acceptability of treatment in } \\
\text { psychotherapy }\end{array}$} \\
\hline & - Subjective rating of the treatment course & \\
\hline & In intervention group: Subjective rating of treatment & \\
\hline
\end{tabular}


adequate diagnostic validity has been reported for social phobia, panic disorder with agoraphobia, agoraphobia without panic disorder, obsessive-compulsive disorder and alcohol abuse/dependence (sensitivity .72-1.00; specificity .63-.80) [29]. Somewhat more modest psychometric properties have been reported for major depressive disorder, generalized anxiety disorder, posttraumatic stress disorder, specific phobia and panic disorder without agoraphobia (sensitivity: .80-.93; specificity: .44-.51) [29].

\section{Suicidal tendencies}

Current suicidal tendencies will be assessed by means of the Suicidal Behaviours Questionnaire-Revised (SBQ-R) [30]. This self-report measure consists of four questions regarding lifetime suicidal tendencies, the frequency of suicidal thoughts during the last 12 months, the course of suicidal intentions, and the probability of suicidal behavior in the future. The SBQ-R has shown high internal consistency. Furthermore, it has shown clinical utility in that scores on the instrument differentiated between subgroups of suicidal and nonsuicidal youth and adults [30].

\section{Anxiety}

Symptoms of anxiety are assessed with the German version of the 7-item Generalized Anxiety Disorder Scale [31] in order to identify patients with a generalized anxiety disorder and to assess anxiety symptoms. The GAD-7 consists of seven items that correspond to the diagnostic criteria for GAD from the DSM-IV. The GAD-7 has good internal consistency and good convergent validity with other anxiety scales [32].

\section{Somatic symptomatology}

To assess the level of somatic symptoms, patients complete the somatic symptom module of the Patient Health Questionnaire (PHQ), the PHQ-15 [33]. The items include the most prevalent somatic symptom complaints reported in primary care. Each symptom is scored from 0 ("not bothered at all") to 2 ("bothered a lot"). The PHQ-15 has shown to be equal or superior to other brief measures for assessing somatic symptoms and screening for somatoform disorders [32].

\section{Quality of life}

To assess quality of life, patients complete the Short-Form Health Survey-12 (SF-12). The SF-12 is based upon the Short-Form Health Survey (SF-36). Its two subscales measure physical and mental aspects of health-related quality of life. It captures general health as well as pain, disabilities in daily life and mental problems. The SF-12 asks for the presence and severity of 12 items over the course of the last four weeks. The re-test reliability is good and it is roughly equivalent to the long form [34].

\section{Psychological aspects of the treatment process}

The Questionnaire for the evaluation of psychotherapeutic progress (FEP-2) is a measure of therapeutic progress and can be used for both change and outcome assessment [35]. Forty items measure the dimensions well-being, symptoms, interpersonal relationships, and incongruence with respect to approach and avoidance goals. The instrument has shown to be change sensitive as well as reliable and it is available in the public domain. It represents the phase model of therapeutic change as well as interpersonal and integrative models of psychotherapy [36].

\section{Psychological empowerment scale}

Empowerment is a psychological motivational construct, which includes for domains: competence, meaning, selfdetermination, and impact. Psychological empowerment will be assessed with an adapted version of the scale by Spreitzer $[37,38]$.

\section{Process measure}

After six and 12 weeks the cooperation between the patient and the therapist will be assessed with the German version of the Working Alliance Inventory - Short (WAI-S; adapted version) [39]. The WAI-short (WAI-S) is a 12item self-report measure of Working Alliance. Each item is rated on a 7-point scale, with higher scores indicating higher alliance. The WAI-S is scored into three subscales measuring Task, Goal and Bond. The Task and Goal scales are intended to measure agreement between the client and therapist with regard to Tasks and Goals of the treatment. The Bond subscale aims to measure the empathic bond between the client and the therapist [40].

\section{Other measurements}

Patients are self-reporting a number of demographic variables including sex, marital status, level of education. Additionally, we assess the patients' medication status.

\section{Therapist measurements}

We collect information from the therapists such as the diagnoses based on clinical judgment, number of therapy sessions, and self-reported usefulness and implementation of deprexis in mental care. Therapists' variables such as age, gender, self-identified theoretical orientation, and years of experience are collected from therapists through a questionnaire.

\section{Statistical analyses}

Primary and secondary outcomes will be conducted on the intention-to-treat (ITT) sample. A linear mixed-model repeated measures ANOVA with time as a within groups factor and treatment condition as a between-groups factor will be used for the main research question. Mixed-model 
repeated measures ANOVA uses all available data of each subject and does not involve the substitution of missing values [41]. With regard to the process variable, i.e., the quality of the working alliance, we will conduct Pearson correlations of the WAI with residualized change scores on the primary and secondary outcomes.

\section{Discussion}

Major depression is a prevalent and debilitating disorder. Even though there is ample evidence for the efficiency and effectiveness of (guided) Internet-based interventions as well as for conventional psychotherapeutic treatments for depression, there are various strengths and limitations for both forms of treatments [11].

In this study, we will examine the effectiveness of psychotherapy plus a web-based online depression program (deprexis) versus traditional psychotherapy in routine care. We expect that depression levels of participants in the intervention group will be significantly lower after three months and after six months compared to the control group. Additionally, we will gain valuable experiences in combining an online psychological intervention with routine psychotherapeutic care.

A major strength of this study is that to the best of our knowledge, it will be the first study that tests whether an adjunctive web-based treatment tool in psychotherapy for depression can be successfully integrated in routine care and whether the combination will show additional effects compared to a regular psychotherapy for depression.

There are important limitations in the present study that should be mentioned: First, we adopted a crossed therapist design, i.e., a given therapist delivers the control as well as the experimental condition. This design is associated with potential drawbacks [42] but was necessary to choose in order to be able to run the study in routine care. In order to adjust for the potential downsides of this design, in the analyses we will control for therapist allegiance. Second, we will not have standardized assessments of depression diagnosis but rely solely on clinical diagnoses of experienced, licensed psychotherapists plus an online self-report diagnostic assessment. Third, in the present study, we will use deprexis as the only adjunctive web-based intervention tool. Therefore, generalizability of the results to other web-based interventions will be restricted.

In addition to the main analysis described in the methods section, our study will be able to address a number of other important research questions, some of which have not been addressed before. For example, we will be able to assess whether an adjunctive web-based treatment affects working alliance scores. Furthermore, we will assess attitudes towards blended psychological interventions from the patients' as well as the therapists' perspective.
If leading to a larger improvement, using effective web-based programs such as deprexis as an adjunctive treatment tool could be an interesting option to consider in future mental health care. Additionally, this study will also give first impressions whether blending face-to-face psychotherapy with a web-based adjunct will have negative side effects, i.e., symptom deterioration or decrease of the patient rated quality of the therapeutic alliance. In general, results of this study will provide an informative basis whether a combination of traditional face-to-face psychotherapy and web-based depression program is feasible and whether it is more effective compared to regular psychotherapeutic treatment in depressed outpatients in routine care.

\section{Trial status}

Currently recruiting $\left(\mathrm{N}_{\text {current }}=28\right.$ as of 800$)$.

\section{Competing interests}

The authors declare that they have no competing interests, with the following exception: BM is employed full-time as research director at Gaia $A G$, the company that developed and owns the deprexis program.

\section{Authors' contributions}

BM, KS, AM and TB contributed to the study design. TK drafted the manuscript. All authors contributed to the further writing of the manuscript. All authors read and approved the final manuscript.

\section{Acknowledgements}

This study is supported by a personal grant to TB from the Swiss National Science Foundation (Switzerland; Ref. PP00P1_144824 /1).

\section{Author details}

${ }^{1}$ Department of Clinical Psychology and Psychotherapy, University of Bern, Bern, Switzerland. ${ }^{2}$ GAIA AG, Hamburg, Germany. ${ }^{3}$ Department of Psychology, City University London, London, United Kingdom. ${ }^{4}$ Deutsche Psychotherapeuten-Vereinigung e.V., DPtV, Berlin, Germany. ${ }^{5}$ Department of Psychopathology and Clinical Intervention, University of Zurich, Zurich, Switzerland.

Received: 30 June 2014 Accepted: 30 September 2014 Published online: 08 October 2014

\section{References}

1. Üstün T, Ayuso-Mateos JL, Chatterji S, Mathers C, Murray CJ: Global burden of depressive disorders in the year 2000. Br J Psychiatry 2004, 184(5):386-392.

2. Richards D, Richardson T: Computer-based psychological treatments for depression: a systematic review and meta-analysis. Clin Psychol Rev 2012, 32(4):329-342

3. Andersson G, Cuijpers P: Internet-based and other computerized psychological treatments for adult depression: a meta-analysis. Cogn Behav Ther 2009, 38:196-205.

4. Cuijpers P, van Straten A, Andersson G, van Oppen P: Psychotherapy for depression in adults: a meta-analysis of comparative outcome studies. J Consult Clin Psychol 2008, 76(6):909-922.

5. Cuijpers P, Donker T, Johansson R, Mohr DC, van Straten A, Andersson G: Self-guided psychological treatment for depressive symptoms: a meta-analysis. PLoS One 2011, 6:e21274.

6. Johansson R, Andersson G: Internet-based psychological treatments for depression. Expert Rev Neurother 2012, 12:861-870.

7. Andersson G, Hesser H, Veilord A, Svedling L, Andersson F, Sleman O, Mauritzson L, Sarkohi A, Claesson E, Zetterqvist V: Randomised controlled non-inferiority trial with 3-year follow-up of internet-delivered versus face-to-face group cognitive behavioural therapy for depression. J Affect Disord 2013, 151(3):986-994. 
8. Titov N, Andrews G, Davies M, Mclntyre K, Robinson E, Solley K: Internet treatment for depression: a randomized controlled trial comparing clinician vs. technician assistance. PLoS One 2010, 5:e10939.

9. Newman MG, Szkodny LE, Llera SJ, Przeworski A: A review of technologyassisted self-help and minimal contact therapies for anxiety and depression: Is human contact necessary for therapeutic efficacy? Clin Psychol Rev 2011, 31(1):89-103.

10. Spek V, Cuijpers P, Nyklícek I, Riper H, Keyzer J, Pop V: Internet-based cognitive behaviour therapy for symptoms of depression and anxiety: a meta-analysis. Psychol Med 2007, 37(3):319-328.

11. Andersson G, Titov N: Advantages and limitations of Internet based interventions for common mental disorders. World Psychiatry 2014, 13(1):4-11.

12. Przeworski A, Newman MG: Palmtop computer-assisted group therapy for social phobia. J Clin Psychol 2004, 60(2):179-188.

13. Newman MG, Kenardy J, Herman S, Taylor CB: Comparison of palmtopcomputer-assisted brief cognitive-behavioral treatment to cognitivebehavioral treatment for panic disorder. J Consult Clin Psychol 1997, 65(1):178-183.

14. Craske MG, Rose RD, Lang A, Welch SS, Campbell Sills L, Sullivan G, Sherbourne C, Bystritsky A, Stein MB, Roy Byrne PP: Computer assisted delivery of cognitive behavioral therapy for anxiety disorders in primary care settings. Depress Anxiety 2009, 26(3):235-242.

15. Carroll K, Ball S, Martino S, Nich C, Babuscio T, Nuro K, Gordon M, Portnoy G, Rounsaville B: Computer-assisted delivery of cognitive-behavioral therapy for addiction: a randomized trial of CBT4CBT. Am J Psychiatry 2008, 165(7):881-888.

16. Carroll KM, Ball SA, Martino S, Nich C, Babuscio TA, Rounsaville BJ: Enduring effects of a computer-assisted training program for cognitive behavioral therapy: a 6-month follow-up of CBT4CBT. Drug Alcohol Depend 2009, 100(1):178-181.

17. Carroll KM, Kiluk BD, Nich C, Gordon MA, Portnoy GA, Marino DR, Ball SA: Computer-assisted delivery of cognitive-behavioral therapy: efficacy and durability of CBT4CBT among cocaine-dependent individuals maintained on methadone. Am J Psychiatry 2014, 171(4):436-444.

18. Wright JH, Wright AS, Salmon P, Beck AT, Kuykendall J, Goldsmith L, Zickel MB: Development and initial testing of a multimedia program for computer-assisted cognitive therapy. Am J Psychother 2002, 56(1):76-86.

19. Wright JH, Wright AS, Albano AM, Basco MR, Goldsmith LJ, Raffield T, Otto MW: Computer-assisted cognitive therapy for depression: maintaining efficacy while reducing therapist time. Am J Psychiatry 2005, 162(6):1158-1164.

20. Månsson KN, Ruiz ES, Gervind E, Dahlin M, Andersson G: Development and initial evaluation of an internet-based support system for face-to-face cognitive behavior therapy: a proof of concept study. J Med Internet Res 2013, 15(12):e280

21. Clough BA, Casey LM: Technological adjuncts to increase adherence to therapy: a review. Clin Psychol Rev 2011, 31(5):697-710.

22. Clough BA, Casey LM: Using SMS reminders in psychology clinics: a cautionary tale. Behav Cogn Psychother 2014, 42(3):257-268.

23. Meyer B, Berger T, Caspar F, Beevers CG, Andersson G, Weiss M: Effectiveness of a novel integrative online treatment for depression (Deprexis): randomized controlled trial. J Med Internet Res 2009, 11(2):e15.

24. Moritz S, Schilling L, Hauschildt M, Schröder J, Treszl A: A randomized controlled trial of internet-based therapy in depression. Behav Res Ther 2012, 50(7):513-521.

25. Berger T, Hämmerli K, Gubser N, Andersson G, Caspar F: Internet-based treatment of depression: a randomized controlled trial comparing guided with unguided self-help. Cogn Behav Ther 2011, 40(4):251-266.

26. Zwarenstein M, Treweek S, Gagnier JJ, Altman DG, Tunis S, Haynes B, Oxman AD, Moher D: Improving the reporting of pragmatic trials: an extension of the CONSORT statement. BMJ 2008, 337:a2390.

27. Cuijpers P, Turner EH, Koole SL, Dijke A, Smit F: What is the threshold for a clinically relevant effect? The case of major depressive disorders Depress Anxiety 2014, 31(5):374-378.

28. Kühner C, Bürger C, Keller F, Hautzinger M: Reliabilität und Validität des revidierten Beck-Depressionsinventars (BDI-II): Befunde aus deutschsprachigen Stichproben. Nervenarzt 2007, 78(6):651-656.

29. Donker T, van Straten A, Marks I, Cuijpers P: A brief Web-based screening questionnaire for common mental disorders: development and validation. J Med Internet Res 2009, 11(3):e19.
30. Osman A, Bagge CL, Gutierrez PM, Konick LC, Kopper BA, Barrios FX: The Suicidal Behaviors Questionnaire-Revised (SBQ-R): validation with clinical and nonclinical samples. Assessment 2001, 8(4):443-454.

31. Spitzer RL, Kroenke K, Williams JB, Löwe B: A brief measure for assessing generalized anxiety disorder: the GAD-7. Arch Intern Med 2006, 166(10):1092-1097.

32. Kroenke K, Spitzer RL, Williams JB, Löwe B: The patient health questionnaire somatic, anxiety, and depressive symptom scales: a systematic review. Gen Hosp Psychiatry 2010, 32(4):345-359.

33. Kroenke K, Spitzer RL, Williams JB: The PHQ-15: validity of a new measure for evaluating the severity of somatic symptoms. Psychosom Med 2002, 64(2):258-266.

34. Ware JE Jr, Kosinski M, Keller SD: A 12-Item Short-Form Health Survey: construction of scales and preliminary tests of reliability and validity. Med Care 1996, 34(3):220-233.

35. Lutz W, Schürch E, Stulz N, Böhnke JR, Schöttke H, Rogner J, Wiedl KH: Entwicklung und psychometrische Kennwerte des Fragebogens zur Evaluation von Psychotherapieverläufen (FEP). Diagnostica 2009, 55(2):106-116.

36. Grawe K: Psychological Therapy. Seattle \& Toronto: Hogrefe Publishing; 2004.

37. Spreitzer GM: Psychological empowerment in the workplace: dimensions, measurement, and validation. Acad Manage J 1995, 38(5):1442-1465.

38. Camerini L, Schulz PJ: Effects of functional interactivity on patients' knowledge, empowerment, and health outcomes: an experimental model-driven evaluation of a web-based intervention. $J$ Med Internet Res 2012, 14(4):e105.

39. Wilmers F, Munder T, Leonhart R, Herzog T, Plassmann R, Barth J, Linster HW: Die deutschsprachige Version des Working Alliance Inventory-short revised (WAI-SR). Ein schulenübergreifendes, ökonomisches und empirisch validiertes Instrument zur Erfassung der therapeutischen Allianz. Klinische Diagnostik Evaluation 2008, 1(3):343-358.

40. Horvath AO, Greenberg LS: Development and validation of the working alliance inventory. J Couns Psychol 1989, 36(2):223-233.

41. Gueorguieva R, Krystal JH: Move over anova: progress in analyzing repeated-measures data audits reflection in papers published in the archives of general psychiatry. Arch Gen Psychiatry 2004, 61(3):310-317.

42. Falkenström F, Markowitz JC, Jonker H, Philips B, Holmqvist R: Can psychotherapists function as their own controls? Meta-analysis of the "crossed therapist" design in comparative psychotherapy trials. J Clin Psychiatry 2013, 74(5):482-491.

doi:10.1186/s12888-014-0285-9

Cite this article as: Krieger et al:: Evaluating an e-mental health program ("deprexis") as adjunctive treatment tool in psychotherapy for depression: design of a pragmatic randomized controlled trial. BMC Psychiatry $201414: 285$

\section{Submit your next manuscript to BioMed Central and take full advantage of:}

- Convenient online submission

- Thorough peer review

- No space constraints or color figure charges

- Immediate publication on acceptance

- Inclusion in PubMed, CAS, Scopus and Google Scholar

- Research which is freely available for redistribution 